\title{
Inadvertent Debridement of the Medial Patellofemoral Ligament Following Arthroscopic Knee Surgery for Infection: A Case Report
}

\author{
Gunjar Jain ${ }^{1}$, Hira Lal Nag ${ }^{1}$, Amit Roy ${ }^{1}$
}

Learning Point of the Article:

The medial patellofemoral ligament injury is a potential complication of arthroscopic debridement of the knee and can lead to habitual dislocation of the patella.

\section{Abstract}

Introduction: Arthroscopic debridement of the knee is usually considered a safe procedure. There are no case reports of medial patellofemoral ligament (MPFL) injury leading to habitual dislocation of the patella following this procedure in the literature.

Case Presentation: A 35-year-old male presented with complaints of habitual dislocation of the left patella, which he noticed after multiple arthroscopic surgeries of the left knee performed elsewhere. He had a history of septic arthritis of the knee following anterior cruciate ligament reconstruction, for which he underwent arthroscopic debridement of the knee twice. After a detailed evaluation, we noticed MPFL medial patellofemoral ligament injury.

Conclusion: The MPFL medial patellofemoral ligament injury occurred as a result of extensive resection of the medial joint capsule while operating for septic arthritis. This rare grave surgical complication emphasizes the care one should take during arthroscopic debridement of the knee.

Keywords: Knee arthroscopy, complications, pyogenic arthritis, patella, dislocation.

\section{Introduction}

Infection following arthroscopic anterior cruciate ligament reconstruction (ACLR) can occur in about $0.1 \%$ to $1.8 \%$ of cases [1]. Because of its rarity, literature is insufficient in this regard, and there are no set guidelines for its management [2]. The superiority of arthroscopic versus open debridement and graft removal versus retention is yet to be established $[3,4,5]$. Arthroscopic debridement of the knee is usually considered a safer procedure compared to open debridement. Most of the literature suggests that the former is associated with lesser operative time, decreased post-operative knee stiffness, and fewer rate of intra-operative and post-operative complications $[4,5]$. However, complications such as Sudeck's osteoarthropathy, graft rupture, and stiffness of the knee have been reported following arthroscopic debridement of the knee $[6,7]$. In the current case, the patient underwent arthroscopic debridement of the knee twice for septic arthritis following ACLR, and subsequently, developed dislocation of the patella. We have not found any reports of such surgical complication in English literature.

\section{Case Presentation}

A 35-year-old male attended the orthopedic outpatient department with complaints of displacement of the left patella as he moves his knee into flexion. He first noticed this symptom 3 months before, when he started mobilization exercise of his left knee following a surgical procedure. He had a history of a twisting injury to his left knee during a road traffic accident 9 months back. He experienced buckling of his left knee following that accident, and a magnetic resonance imaging (MRI) showed a torn anterior cruciate ligament (ACL) at the midsubstance level. After 2 months, he underwent an arthroscopic

\section{Author's Photo Gallery}

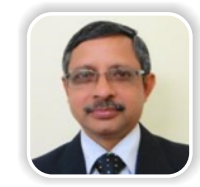

Dr. Hira Lal Nag

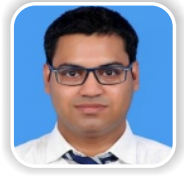

Dr. Amit Roy

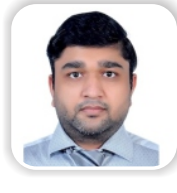

Dr. Gunjar Jain
DOI:

10.13107/jocr.2021.v11.i03.2102
${ }^{1}$ Department of Orthopaedics, All India Institute of Medical Sciences, New Delhi. India.

Address of Correspondence:

Dr. Gunjar Jain,

Department of Orthopaedics, All India Institute of Medical Sciences, House No. 340, Masjid Moth, New Delhi - 110 049. India.

E-mail: drgunjarjain@gmail.com

Journal of Orthopaedic Case Reports | pISSN 2250-0685 | eISSN 2321-3817 | Available on www.jocr.co.in | doi:10.13107/jocr.2021.v11.i03.2102 This is an Open Access article distributed under the terms of the Creative Commons Attribution Non-Commercial License (http://creativecommons.org/licenses/by-nc/3.0) which permits unrestricted non-commercial use, distribution, and reproduction in any medium, provided the original work is properly cited. 


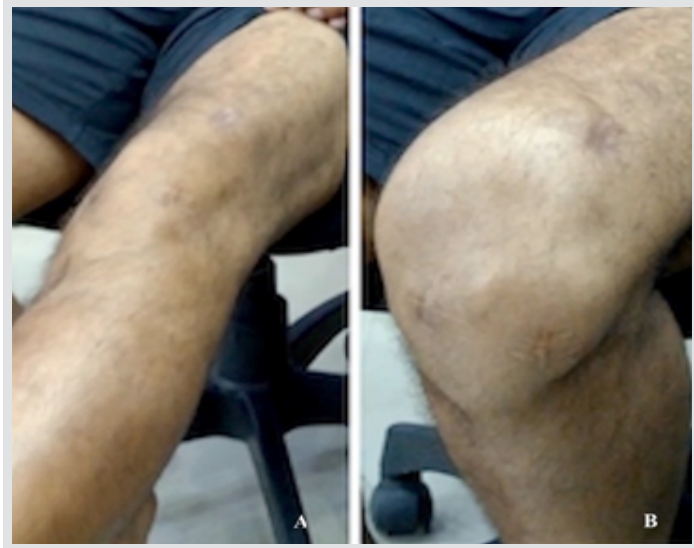

Figure 1: Clinical photograph of the left knee showing the patella reduced in place, (a) while the knee is in extension, and laterally dislocated patella, (b) while the knee is in flexion. The scar marks of previous surgeries can also be seen.

ACLR using the ipsilateral semitendinosus autograft in a local hospital.

The patient developed discharge from the surgical wound site associated with fever on the seventh postoperative day. The inflammatory markers, including the erythrocyte sedimentation rate, $\mathrm{C}$-reactive protein, and the total leukocyte count, were elevated. Finally, he got diagnosed with septic arthritis, and arthroscopic debridement of the knee was done in the same hospital, preserving the graft. The condition got resolved initially, and the patient began the mobilization exercises with gradual improvement. However, the features of septic arthritis reappeared 3 months later. The patient underwent a repeat arthroscopic debridement with removal of the graft after 2 weeks of the onset of this second episode of infection. The patient began the range of motion exercise on the second day of this surgery and noticed the patella is dislocating every time he flexes his knee.

On clinical examination, we found dislocation of the patella on each cycle of flexion of the knee, which reduces spontaneously on the extension (Fig. 1). Since, unlike recurrent dislocation, the patella was relocating on the extension without any pain and swelling, we termed it a habitual dislocation of the patella. However, there were no signs of active infection. The surgical scar marks of previous procedures were observed, including scars of superomedial, and superolateral, portals. Furthermore, there was no generalized hyperlaxity or exaggeration of anteversion of the femur. The Q-angle was $15^{\circ}$. The skyline view radiograph of both the knees did not indicate trochlear dysplasia considering the sulcus angle on the left side, which was $130^{\circ}$ (Fig. 2). The Insall Salvati ratio measured by dividing patellar tendon length to patellar height was 0.8 .

On further evaluation, we found the tibial tuberosity-trochlear groove (TT-TG) distance to be $18.4 \mathrm{~mm}$ (Fig. 3), which indicates a mild distal malalignment that predisposes the patient to dislocation of the patella. We performed an MRI of the left knee and noticed a medial patellofemoral ligament (MPFL) tear (Fig. 4a and b). The MPFL must have been injured during the surgery, as the patient experienced his first dislocation immediately after the second arthroscopic debridement procedure and as the initial MRI performed

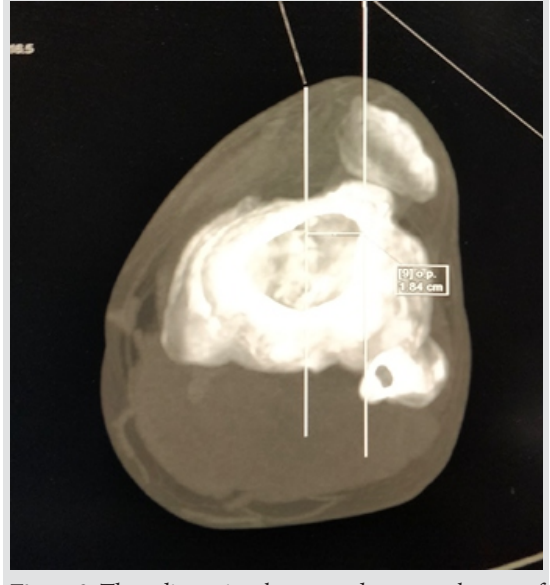

Figure 3: Three dimensional computed tomography scan of the left knee with superimposed trochlear groove and the tibial tuberosity demonstrating the tibial tuberositytrochlear groove distance to be $1.84 \mathrm{~cm}$.
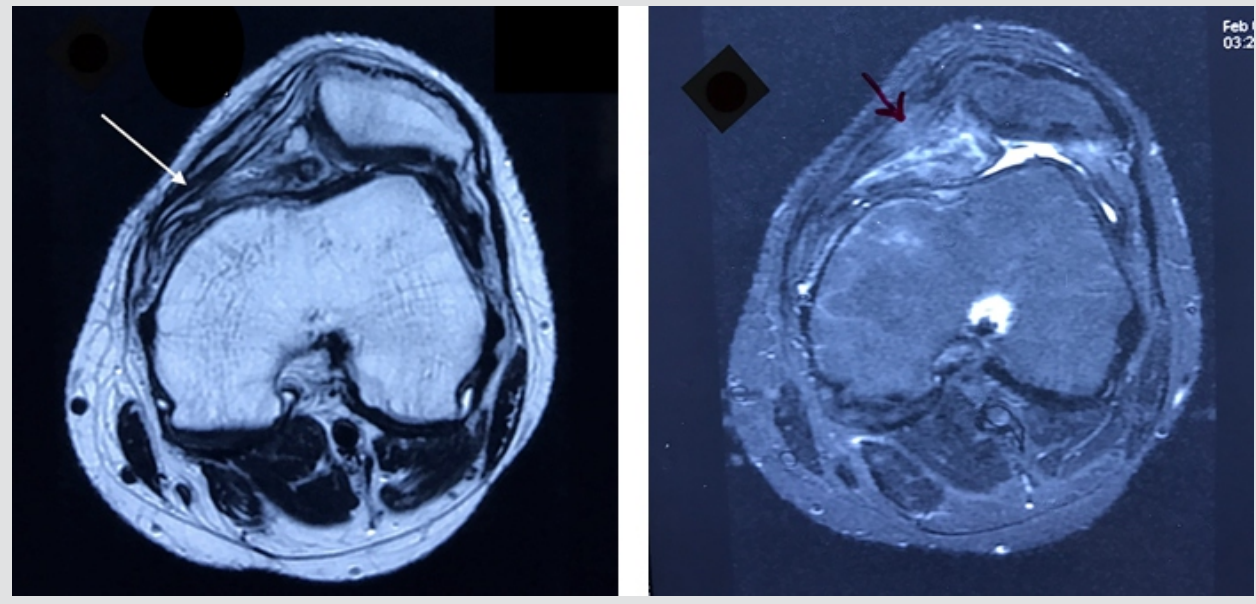

Figure 4: (a) T1- weighted magnetic resonance imaging axial section of the left knee demonstrating injury to the Medial patellofemoral ligament (arrow). (b) T2- weighted magnetic resonance imaging axial section of the left knee demonstrating injury to the medial patellofemoral ligament (arrow). 


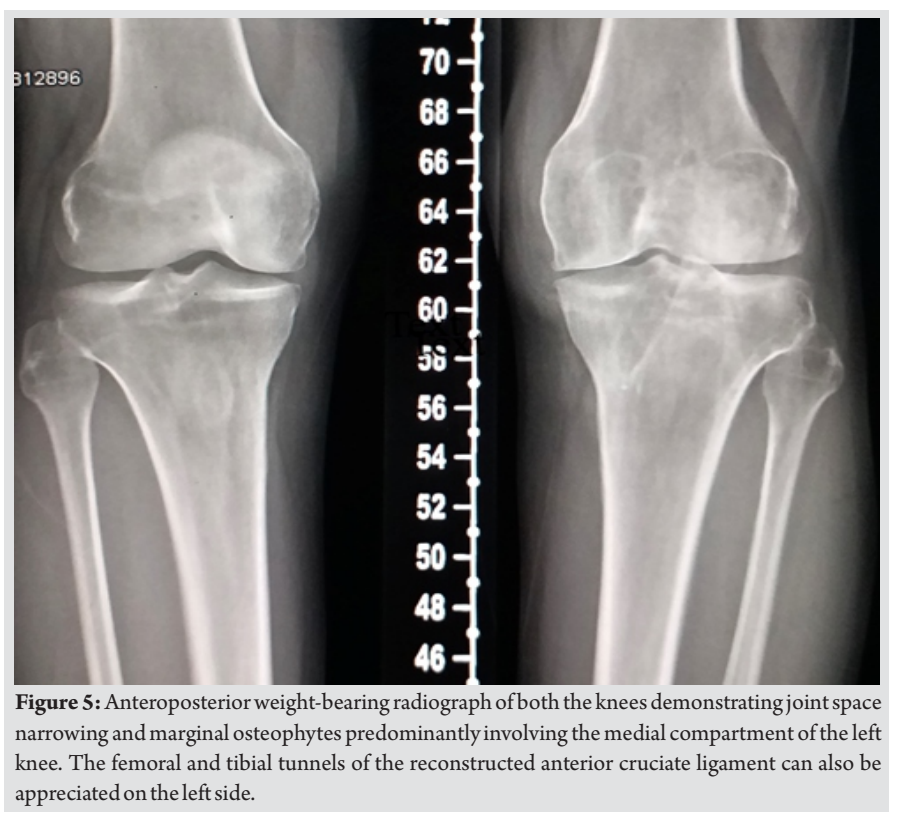

before the ACL reconstruction suggested an intact MPFL. Therefore, we made a diagnosis of iatrogenic MPFL tear.

For the habitual dislocation of the patella, we planned an MPFL reconstruction using the contralateral semitendinosus autograft. Despite explaining the need for surgical intervention, the patient denied any surgical procedure and preferred nonoperative management. At 2 years of follow-up, the patient does not have any pain in his left knee and complaints of only mild buckling while walking on uneven ground. However, the habitual dislocation of the patella persists. Nevertheless, the patient performs his daily pursuits, including walking, squatting, and climbing stairs without much difficulty. There are no features suggestive of any active infection. The recent radiograph shows signs of early osteoarthritic changes (Fig. 5). The patient is still unwilling for any surgery and is under our regular follow-up.

\section{Discussion}

Arthroscopic debridement of the knee is done in conditions such as septic arthritis following ACLR, primary septic arthritis, and osteoarthritis of the knee. Most of the surgeons regard arthroscopic debridement as a reliable procedure with very low complications. However, in this case, iatrogenic injury to the MPFL ligament occurred, which lead to habitual dislocation of the patella. There are no reports in the literature concerning MPFL injury following arthroscopic debridement of the knee. Hence, we want to bring notice of this grave complication to the arthroscopic surgeons and prevent any such catastrophe in their practice.

In this case of ACLR complicated by septic arthritis, the primary surgeons removed the graft after the persistence of the infection despite an initial arthroscopic debridement. Hantes et al. obtained good to excellent outcomes following a similar approach in the management of their cases [8]. Most of the authors prefer to retain the graft in case of septic arthritis following ACLR $[2,9]$. A recent literature review shows that $43.8 \%$ of the patients who underwent graft removal required a secondary ACLR, but only $6.5 \%$ of patients with graft retention required this second surgery [2]. Surprisingly, however, the expected value decision analysis recommends graft removal as the preferred treatment as most of the patients would prefer to avoid a late reoperation [2].

\section{Conclusion}

This case is an original case report of interest to orthopedics, particularly to arthroscopic surgeons. It describes the development of habitual dislocation of the patella following arthroscopic debridement of the knee. This unforeseen and grave development occurred as a result of extensive resection of the joint capsule on the medial side, which damaged the MPFL.

\section{Clinical Message}

This case highlights an unusual complication, i.e., habitual dislocation of the patella after arthroscopic debridement of the knee. Such a complication has never been described in the literature and thus, a piece of new information. The primary takeaway information from this case report is to acknowledge this devastating complication and follow adequate care during arthroscopic debridement of the knee.

\section{References}

1. Wang C, Lee YH, Siebold R. Recommendations for the management of septic arthritis after ACL reconstruction. Knee Surg Sports Traumatol Arthrosc 2014;22:2136-44.

2. Kusnezov N, Eisenstein ED, Dunn JC, Wey AJ, Peterson DR, Waterman BR. Anterior cruciate ligament graft removal versus retention in the setting of septic arthritis after reconstruction: A systematic review and expected value decision analysis. Arthrosc J Arthrosc Relat Surg
2018;34:967-75.

3. Bovonratwet P, Nelson SJ, Bellamkonda K, Ondeck NT, Shultz BN, Medvecky MJ, et al. Similar 30-day complications for septic knee arthritis treated with arthrotomy or arthroscopy: An American college of surgeons national surgical quality improvement program analysis. Arthrosc J Arthrosc Relat Surg 2018;34:213-9.

4. Johnson DJ, Butler BA, Hartwell MJ, Fernandez CE, Nicolay 
RW, Selley RS, et al. Arthroscopy versus arthrotomy for the treatment of septic knee arthritis.J Orthop 2020;19:46-9.

5. Saper M, Stephenson K, Heisey M. Arthroscopic irrigation and debridement in the treatment of septic arthritis after anterior cruciate ligament reconstruction. Arthrosc J Arthrosc Relat Surg 2014;30:747-54.

6. Bert JM, Bert TM. Management of infections after arthroscopy. Sports Med Arthrosc Rev 2013;21:75-9.

7. Abdel-Aziz A, Radwan YA, Rizk A. Multiple arthroscopic debridement and graft retention in septic knee arthritis after ACL reconstruction: A prospective case-control study. Int Orthop 2014;38:73-82.

8. Hantes ME, Raoulis VA, Doxariotis N, Drakos A, Karachalios T, Malizos KN. Management of septic arthritis after arthroscopic anterior cruciate ligament reconstruction using a standard surgical protocol. Knee 2017;24:588-93.

9. Otchwemah R, Naendrup JH, Mattner F, Tjardes T, Bäthis H, Shafizadeh S. Effective graft preservation by following a standard protocol for the treatment of knee joint infection after anterior cruciate ligament reconstruction.J Knee Surg 2019;32:1111-20.

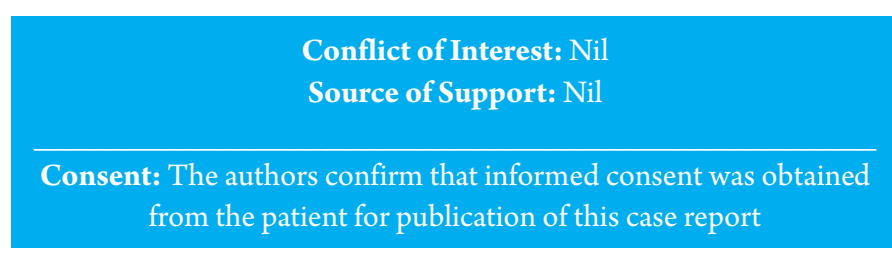

\section{How to Cite this Article}

Jain G, Nag HL, Roy A. Inadvertent Debridement of the Medial Patellofemoral Ligament Following Arthroscopic Knee Surgery for Infection: A Case Report. Journal of Orthopaedic Case Reports 2021 March;11(3): 90-93. 